Research

Open Access

\title{
Outcome after severe head injury: focal surgical lesions do not imply a better Glasgow Outcome Score than diffuse injuries at 3 months
}

\author{
Paul Leach ${ }^{1}$, Omar N Pathmanaban*1, Hiren C Patel ${ }^{1}$, Julian Evans ${ }^{1}$, \\ Raphael Sacho ${ }^{1}$, Richard Protheroe ${ }^{2}$ and Andrew T King ${ }^{1}$
}

Address: ${ }^{1}$ Division of Neurosurgery, GMNC, Manchester Academic Health Sciences Centre, Salford Royal NHS Foundation Trust, University of Manchester, Manchester, UK and 2Department of Neurointensive Care, GMNC, Manchester Academic Health Sciences Centre, Salford Royal NHS Foundation Trust, University of Manchester, Manchester, UK

Email: Paul Leach - paulleach@live.co.uk; Omar N Pathmanaban* - omar.pathmanaban@manchester.ac.uk;

Hiren C Patel - hiren.patel@srft.nhs.uk; Julian Evans - julianevans@doctors.org.uk; Raphael Sacho - raphael.sacho@postgrad.manchester.ac.uk; Richard Protheroe - richard.protheroe@srft.nhs.uk; Andrew T King - andrew.king@srft.nhs.uk

* Corresponding author

Published: 3 April 2009

Journal of Trauma Management \& Outcomes 2009, 3:5 doi:10.1186/1752-2897-3-5

This article is available from: http://www.traumamanagement.org/content/3/I/5

(c) 2009 Leach et al; licensee BioMed Central Ltd.

This is an Open Access article distributed under the terms of the Creative Commons Attribution License (http://creativecommons.org/licenses/by/2.0), which permits unrestricted use, distribution, and reproduction in any medium, provided the original work is properly cited.
Received: 20 November 2008

Accepted: 3 April 2009

\begin{abstract}
Background: Historically neurosurgeons have accepted head injured patients only in the presence of a mass lesion requiring surgical decompression. Underpinning this is an assumption that these patients have a better outcome than patients without a surgical lesion. This has meant that many patients without a surgical lesion have been managed locally in the referring hospital. However, there is now evidence that treatment of all head injured patients in a specialist centre leads to improved outcomes. Therefore, we have asked the question: does the presence of a surgical lesion imply better outcome from severe head injury?
\end{abstract}

Results: We prospectively recorded the Glasgow Outcome score (GOS), at 3 months, of all the severely head injured patients treated at our institution over a two and a half year period. Of 116 patients admitted with an initial Glasgow Coma Score (GCS) of 8 or less, 58 had surgical lesions and 58 non-surgical head injuries. The two groups were well matched for presenting GCS and age. Overall our favourable outcome rate (GOS 4 and 5) at 3-months for the patients with a surgical lesion and for the non-surgical group were $47.3 \%$ and $46.6 \%$ respectively, with no significant difference between the two $(P=0.54)$.

Conclusion: The assumption in the past has always been that patients presenting in coma from traumatic diffuse brain injury will do worse than those that have a mass lesion amenable to surgical decompression. Our series would suggest that this is not the case and all severely head injured patients should expect similar outcome when cared for in a neuroscience centre.

\section{Background}

It is well accepted that prompt surgical decompression of traumatic intracranial haematomas leads to improved outcomes for head injured patients. [1-3] In keeping with this, recent prognostic models have identified non-evacuated intracranial haematomas as strong predictors of poor outcome in severe head injured patients.[4] As such, it is usual for patients with traumatic intracranial mass lesions 
Table I: Mean ages and median initial presenting GCS of the surgical and non-surgical groups

\begin{tabular}{cccc}
\hline & Surgical group & Non-surgical group & $\begin{array}{c}\text { Statistical difference } \\
\text { (P-Value) }\end{array}$ \\
\hline Mean age (years) & 39.1 (range 16-69) & 33.3 (range 16-78) & 0.05 \\
\hline Median initial GCS & 4 & 5 & 0.66 \\
\hline
\end{tabular}

to be transferred to a neurosciences centre for surgery and neurointensive care.

Conversely, due to lack of infrastructure, patients deemed to have non-surgical head injuries have often been managed outside of neuroscience centres. This approach to allocating limited resources is based on a long-held belief that individuals with focal surgical lesions will do better than those with diffuse injuries. Indeed, contemporary data predicts that individuals with extradural haematomas (EDH) will do better than patients with other types of severe closed head injury.[5] This is intuitive because an EDH compromises neural function by compression, but often has minimal associated underlying parenchymal injury; prompt surgical evacuation will thus lead to a good recovery.

However, biased resource allocation in favour of the surgical group is now controversial because it has been shown that the non-surgical group of severely head injured patients also fare better by treatment in a specialist centre.[6] Moreover, the prognostic advantage for EDH should not necessarily be extrapolated to other traumatic mass lesions such as acute subdural haematomas or contusions, where associated parenchymal injury is a usual feature.

Despite this, there remains a paucity of evidence in the literature on the expected outcome of severely head injured patients without a mass lesion as compared to those with a surgical lesion when both groups are treated in a special-

Table 2: Glasgow Outcome Scores at 3-months for the patients requiring surgical decompression treated in our unit over the study period

\begin{tabular}{ccc}
\hline GOS & Number of patients & $\%$ \\
\hline 1 & 18 & 31.6 \\
\hline 2 & 2 & 3.5 \\
\hline 3 & 10 & 17.5 \\
\hline 5 & 17 & 29.8 \\
\hline
\end{tabular}

ist centre. Therefore, we have compared our outcomes for severely head injured patients with and without mass lesions.

\section{Results}

Study Cohorts

Over the study period 116 patients with an initial GCS of eight or less were admitted to Greater Manchester Neuroscience Centre. Ninety one patients were male and 25 female. The age range was $16-78$ with the mean age being 36.2 years. Of these 116 patients, 58 had a mass lesion that required surgical evacuation and 58 had a non-surgical head injury. The age and presenting GCS of these two groups were compared using an independent sample 2tailed t test and the Mann-Whitney $U$ test respectively. The two cohorts were well matched for presenting GCS with no significant difference $(P=0.66)$. There was a statistically significant difference in age $(P=0.05)$, but not a biologically significant difference (see table 1 ).

\section{Glasgow Outcome Scores (GOS) at 3 months}

Outcome data was collected for 115 patients as 1 patient in the surgical group was lost to follow up. The GOS at 3months for the surgical group and for the non-surgical group are shown in tables 2 and 3 respectively. Our favourable outcome rate (GOS 4 and 5) at 3-months for the patients with a surgical lesion and for the non-surgical group were $47.3 \%$ and $46.6 \%$ respectively. To assess any difference in outcome between the surgical and non-surgical cohorts, we applied a Mann-Whitney U test to the dataset after dichotomising GOS into good (GOS 4-5)

Table 3: Glasgow Outcomes Scores at 3-months for the patients with non-surgical severe head injury treated in our unit over the study period

\begin{tabular}{ccc}
\hline GOS & Number of patients & $\%$ \\
\hline 1 & 22 & 37.9 \\
\hline 2 & 1 & 1.7 \\
\hline 3 & 8 & 13.8 \\
\hline 5 & 19 & 32.8 \\
\hline
\end{tabular}


Table 4: Favourable outcomes (\%) achieved in three different series of severely head injured patients prior to the institution of protocol driven therapy

\begin{tabular}{|c|c|c|c|}
\hline Author & Epoch & Number of patients & Favourable outcome \\
\hline Fakhry [9] et al & $1991-1994$ & $N=219$ & $43.3 \%$ \\
\hline Patel $[10]$ et al & $1991-1993$ & $N=53$ & $40.4 \%$ \\
\hline Warme $[\mathrm{II}]$ et al & $1980-198 \mid$ & $N=49$ & $32 \%$ \\
\hline
\end{tabular}

and poor (GOS 1-3) outcomes. There was no significant difference in outcome between the two groups $(\mathrm{P}=0.54)$.

\section{Discussion}

We have demonstrated that a favourable outcome (GOS 4 or 5) for patients with non-surgical severe traumatic brain injury $(46.6 \%)$ was not significantly different to the outcome for patients in the surgical cohort $(47.3 \%)(\mathrm{P}=$ $0.54)$. There are few studies that have explicitly and prospectively assessed outcome in patients with severe nonsurgical head injury and therefore our results serve as a good reference point. In the Traumatic Coma Database study group [8] 85\% of patients with diffuse injury had a poor outcome at hospital discharge which is significantly poorer than our results at three months. On the other hand, the overall outcome from other contemporary series might suggest that our outcomes fall short of the results from the 'best centres'. At the time of this study, our institution did not have a protocol driven head injury management scheme and in light of this, these results compare favourably with those reported from the pre-protocol era (see Table 4).

Historically neurosurgeons have accepted patients with major head injuries only in the presence of a mass lesion requiring surgical decompression. They have been accused of "cherry picking" and their good results may be attributed to this. It has recently been suggested that this approach is not appropriate as patients treated in a nonneurosurgical setting with a diffuse injury have a higher probability of death compared with those treated in a neurosurgical setting.[6] The role of neurosurgical care is further supported by the observation of higher mortality in patients operated on for an acute subdural haematoma who were transferred back to the ICU at the referring hospital due to lack of speciality beds.[12] What is more, Varelas et al found that the appointment of a neurointensivist to their neurosurgical ICU improved the outcomes of their head injured patients.[13]

Finally, there is a long held argument that aggressive, or specialist facilities lead to increased survival, but with a resultant increment in the number of patients left with severe disability or in a persistent vegetative state (PVS).
The results here demonstrate in line with others that the PVS rate in patients with diffuse injury is very low.

\section{Conclusion}

The assumption in the past has always been that patients presenting in coma from traumatic diffuse brain injury will do worse than those that have a mass lesion amenable to surgical decompression. Our series would suggest that this is not the case and all severely head injured patients should expect a similar outcome. Admission to a neuroscience centre impacts favourably on outcome and our data suggests the absence of a lesion requiring surgical decompression does not imply a poorer outcome. Therefore the authors are of the opinion that severely head injured people should be managed in a specialist centre regardless of the presence of a surgical lesion.

\section{Methods}

Outcome data was collected at three months post injury for all patients with a head injury, presenting initially with a Glasgow Coma Score (GCS) of eight or less, admitted to our intensive care unit (ICU), over a two and a half year period (July 2003 - December 2005). The initial presenting GCS was taken as the first documented GCS from the time of injury. Data was recorded within 24 hours of admission to our unit as part of a routine auditing process. There was no specific admission policy to our unit for these patients over the study period. Patients were admitted to the unit at the discretion of the on-call Consultant Neurosurgeon. They were admitted for either decompression of a mass lesion or medical management of their intracranial pressure (ICP). Patients or relatives were contacted at 3 months via telephone and assessed by the lead author (PL) using the Glasgow Outcome Score (GOS).[7] Outcomes of patients requiring surgical decompression for a mass lesion were compared to the outcomes for the non-surgical group.

\section{Competing interests}

The authors declare that they have no competing interests.

\section{Authors' contributions}

PL and AK were responsible for the conception and design of the study. PL collected all data. All authors contributed 
to the analysis and interpretation of data which was initially performed by OP, PL and RS. OP and PL drafted the manuscript and all authors reviewed it critically for intellectual content and have given final approval of the version to be published.

\section{References}

I. Wester K: Decompressive surgey for "pure" epidural hematomas: does neurosurgical expertise improve the outcome? Neurosurgery 1999, 44(3):495-500.

2. Haselsberger K, Pucher R, Auer LM: Prognosis after acute subdural or epidural haemorrhage. Acta Neurochir 1988, 92:III.

3. Seelig JM, Becker DP, Miller JD, et al.: Traumatic acute subdural haematoma. NEJM 1981, 304(25): I5I I-1517.

4. MRC CRASH Trial Collaborators, Perel P, Arango M, Clayton T, et al.: Predicting outcome after traumatic brain injury: practical prognostic models based on large cohort of international patients. BMJ 2008, 336(764I):425-9.

5. Steyerberg EW, Mushkudiani N, Perel P, et al.: Predicting outcome after traumatic brain injury: development and international validation of prognostic scores based on admission characteristics. PLoS Med 2008, 5(8):el65.

6. Patel HC, Bouamra $O$, Woodford $M$, et al: Trends in head injury outcome from 1989 to 2003 and the effect of neurosurgical care: an observational study. Lancet 2005, 366(9496): I538-44.

7. Jennett $B$ : Assessment of outcome after severe brain damage. A practical scale. Lancet 1975, I(7905):480-4.

8. Marshall LF, Gautille T, Klauber, et al.: The outcome of severe closed head injury. J Neurosurg |991, 75(5):S28-S36.

9. Fakhry SM, Trask AL, Waller MA, et al.: Management of braininjured patients by an evidence-based medicine protocol improves outcomes and decreases hospital charges. J Trauma 2004, 56(3):492-9.

10. Patel HC, Menon DK, Tebbs S, et al.: Specialist neurocritical care and outcome from head injury. Intensive Care Med 2002, 28(5):529-3I.

II. Warme PE, Bergstrom R, Persson L: Neurosurgical intensive care improves outcome after severe head injury. Acta Neurochir 1991, II 10(I-2):57-64.

12. Jaeger M, Schuhmann MU, Samii M, et al.: Neurosurgical emergencies and missing neurosurgical intensive care unit capacity: is "operate-and-return" a sound policy? E J Emerg Med 2002, 9(4):334-338.

13. Varelas PN, Eastwood D, Yun HJ, et al.: Impact of a neurointensivist on outcomes in patients with head trauma treated in a neurosciences intensive care unit. J neurosurg 2006, 104(5):713-9.
Publish with BioMed Central and every scientist can read your work free of charge

"BioMed Central will be the most significant development for disseminating the results of biomedical research in our lifetime. "

Sir Paul Nurse, Cancer Research UK

Your research papers will be:

- available free of charge to the entire biomedical community

- peer reviewed and published immediately upon acceptance

- cited in PubMed and archived on PubMed Central

- yours - you keep the copyright

Submit your manuscript here:

http://www.biomedcentral.com/info/publishing_adv.asp
BioMedcentral 